\title{
CARGA FINANCIERA DEL CUIDADO DEL ENFERMO CRÓNICO EN UN GRUPO DE FAMILIAS EN GUATEMALA
}

\section{THE FINANCIAL BURDEN OF FAMILY CARE OF THE CHRONICALLY ILL IN GUATEMALA}

\section{TITULO CORTO: CARGA FINANCIERA DEL CUIDADO DEL ENFERMO CRÓNICO}

\author{
Orfa Nineth Morales-Padilla ${ }^{1}$, Gladys Ayde Pinituj-Monroy ${ }^{2}$, Crisanda Alcira Escobar-Vásquez ${ }^{3}$
}

Recibido en marzo 11 de 2016

Aceptado en junio 28 de 2016

\section{RESUMEN}

Como objetivo del trabajo, se planteó describir la carga financiera del cuidado del enfermo crónico en un grupo de familias en Guatemala, para ello se realizó un estudio descriptivo, de corte transversal que se desarrolló en 2014 como parte de un estudio multicéntrico. Se emplearon dos encuestas para la recolección de datos: Para caracterizar al grupo familiar la encuesta "GCPC-UN- D y para conocer el consumo real efectivo de las familias la encuesta "Costo financiero del cuidado de la Enfermedad crónica”. Para analizar la carga financiera se empleó la Metodología CARACOLA. Los resultados revelaron que los costos propios de salud, seguidos por los de transporte, vivienda, comunicaciones y alimentos son los que en conjunto y en ese orden producen carga financiera familiar del cuidado del enfermo crónico. Se concluye que el consumo real efectivo de un grupo de familias en Guatemala asociado con el cuidado de un enfermo crónico, genera carga financiera elevada produciendo un impacto importante en la estabilidad familiar y dificulta aún más la experiencia de salud. Es clara la necesidad de incluir en las políticas públicas la forma de considerar y mitigar los costos del cuidado

Palabras clave: Enfermedad crónica; Costo de enfermedad; Economía de la salud; Guatemala

\section{ABSTRACT}

The aim of research was to describe and analyze the financial burden of family care of the chronically ill Guatemala. It was developed throught a descriptive, cross-sectional study that was development in 2014 as part of multicenter study. The Instruments "GCPC-UN- D" were used to characterize the subjects and the Survey Financial cost of chronic disease care, to identify the real effective household consumption. The financial burden attributable to family care

1. Magister en Enfermería. Hospital Nacional de Ortopedia y Rehabilitación Dr. Jorge Von Ahn. Ciudad de Guatemala, Guatemala. Correo: publicacion.enf.INC@ gmail.com: http://orcid.org/0000-0003-4445-0102

2. Licenciada en Enfermería. Hospital Nacional de Ortopedia y Rehabilitación Dr. Jorge Von Ahn. Ciudad de Guatemala, Guatemala. Correo: gladysaydepinitujmonroy@ hotmail.com

3. Licenciada en Enfermería. Hospital Nacional de Ortopedia y Rehabilitación Dr. Jorge Von Ahn. Ciudad de Guatemala, Guatemala. Correo: enfriaorto@hotmail.com

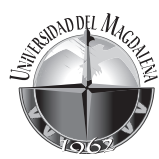


was determined under the CARACOLA methodology, which quantifies the level of the burden and financial cost of it and guides describe the attributes of household consumption associates with patient care. The results revealed that own health costs, followed by transport, housing communications and food are those that together and in that order produce family financial burden of care of the chronically ill in a group of families in Guatemala. It concludes that family group in Guatemala has a high financial burden attributable to caring for a person with chronic disease. It produces a significant impact on family stability and even more difficult health experience. Is a clear need for public policy include how to consider and mitigate the costs of care.

Keywords: Cost of Illness; Chronic disease; Health economies; Guatemala

\section{INTRODUCCIÓN}

Según la Organización Mundial de la Salud (OMS), las Senfermedades crónicas (EC) son de larga duración y de lenta evolución y están generando uno de los mayores problemas de salud pública que hay en el mundoํ.

Para Guatemala, la OMS señala que el total de muertes de todas las edades en ambos sexos se distribuyen: 14\% de enfermedad cardiovascular, el $12 \%$ de diversos tipos de cáncer, el $3 \%$ de enfermedades respiratorias crónicas, el $5 \%$ de diabetes, $14 \%$ otras EC, $34 \%$ entre enfermedad contagiosa, materna, perinatal y de origen nutricional y $18 \%$ de lesiones, es decir, casi la mitad de las muertes (48\%) se asocian con la $\mathrm{EC}^{2}$. Quizás por el impacto que genera la EC en el paciente y en su grupo familiar y social, su incremento es mundialmente reconocido como un problema prioritario.

De acuerdo con la literatura, los costos familiares que se tienen cuando se enfrenta el cuidado de alguno de sus miembros se asocia con falta de acceso real a la seguridad social y atención en salud ${ }^{3}$ con factores socioeconómicos relacionados con gastos propios de salud ${ }^{4}$.

La presencia de enfermedad crónica en uno de los integrantes del grupo familiar es una gran amenaza para la estabilidad económica del grupo ${ }^{5}$. Son las familias las que viven la mayor parte del tiempo cuidando a las 46 personas enfermas ${ }^{6}$ y muchas de ellas entran en círculos de enfermedad y pobreza ${ }^{6}$. Más aún, algunas de estas dificultades se agudizan en sectores rurales ${ }^{7}$. Por ello, conocer para poder aliviar la carga financiera que tienen las familias ${ }^{8}$, es una prioridad, en especial al considerar la forma exponencial en que está incrementando la $\mathrm{EC}^{1}$. En América Latina se han adelantado diferentes estudios de carga de la EC, donde se valoran los años de vida saludable perdidos o años de vida ajustados por discapacidad (AVISAS) ${ }^{9,10}$. En esta misma región, la familia es un referente fundamental para enmarcar el cuidado de la salud. Familias que cuidan a un familiar con EC han reportado percepción de carga asociada con la falta de conocimiento y habilidad para abordar la situación ${ }^{11-12}$, que coincide con estudios de otras latitudes donde se refleja alteración en la calidad de vida, en especial del cuidador principal ${ }^{13}$.

En el estudio de disminución de la carga de la EC en Colombia, se analizó la proporción en que los asuntos financieros preocupaban a los cuidadores familiares encontrando que el $89 \%$ de ellos la refiere y que se asocia con ingresos insuficientes y dificultad para responder por las necesidades familiares básicas como el pago de alquiler, los gastos de alimentación, el pago de deudas, los gastos en salud, el pago de servicios públicos y el transporte ${ }^{14}$. A pesar de ello, este componente financiero ha sido poco abordado en la región y no se conocen estudios al respecto en Guatemala. El objetivo del estudio fue conocer y analizar los diferentes aspectos del costo financiero del cuidado familiar de un grupo de personas con EC en Guatemala.

\section{MATERIALES Y MÉTODOS}

El estudio tuvo un diseño descriptivo, de corte transversal y tal como se señaló hace parte de una investigación multicéntrica coordinada por el Programa para la Disminución de la Carga de la EC en Colombia.

Su muestra fue intencional y consistió en la selección de 30 familias trabajadoras del Hospital Nacional de Ortopedia y Rehabilitación. 
Se emplearon para la recolección de datos dos encuestas aplicadas en las familias, a la diada (persona con enfermedad crónica- cuidador familiar). Para caracterizar al grupo familiar la encuesta "GCPC-UN-D", que contiene 42 ítems y tres dimensiones: las condiciones y perfil socio demográfico de la Diada cuidador familiar-paciente, la percepción de carga y apoyo y los medios de información y comunicación con que cuentan. Esta encuesta ha sido validada para el contexto de América Latina ${ }^{15,16}$. Para conocer el consumo real efectivo de las familias la encuesta "Costo financiero del cuidado de la Enfermedad crónica" de Montoya et $\mathrm{al}^{17}$ que permite determinar el consumo real efectivo familiar y el nivel de carga que dicho consumo genera.

Para analizar la carga financiera se empleó la Metodología CARACOLA $^{18}$ (Carga financiera atribuible al cuidado familiar de una persona con enfermedad crónica en América Latina), que cuenta con dos facetas articuladas: Cuantificación del nivel de la carga y costo financiero de la misma. La primera tiene como objeto identificar el nivel del impacto o peso relativo del costo financiero en cada uno de los rubros o agrupación de ellos y la segunda se orienta a describir los atributos peculiares del consumo del grupo familiar asociado al cuidado del paciente. Inicialmente esta metodología se probó en Colombia y se denominó CARACOL (Carga financiera atribuible al cuidado familiar de una persona con enfermedad crónica en Colombia). Tal como fue descrita por sus autores, para concretar el peso relativo de la carga, se determina que por $\mathrm{C}_{\mathrm{Xji}} \mathrm{C}_{\mathrm{Xji}}$ y $\mathrm{C}_{\mathrm{Cji}} \mathrm{C}_{\mathrm{Cji}}$ se denota respectivamente, la cantidad de dinero que la familia i consume, $(\mathrm{i}=1,2$, ...,n) en el cuidado del enfermo y la cantidad de dinero que la familia consumía antes de que se presentara la enfermedad en un determinado rubro $\mathrm{R}_{\mathrm{j}}$ de la economía doméstica, $\mathrm{j}=1,2, \ldots, \mathrm{k}$, siendo $\mathrm{n}$ el tamaño de la muestra, es decir, el número de familias elegidas para el estudio, y k el número total de rubros considerados en el mismo. El cociente de las dos cantidades.

$$
t_{j i}=\frac{C_{X_{j i}}}{C_{C_{j i}}}
$$

Expresa la proporción del consumo atribuible a la enfermedad respecto al consumo familiar en el rubro específico $\mathrm{R}_{\mathrm{i}}$, independiente del valor monetario (Figura 1).

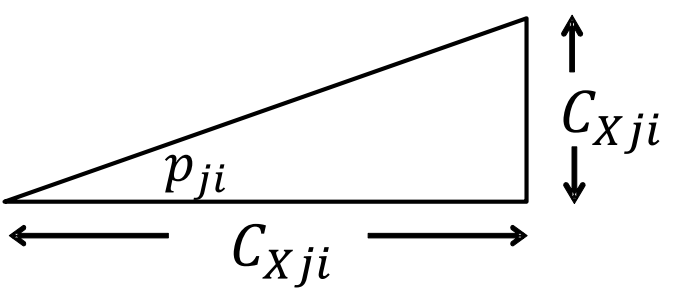

Figura 1. Orientación del cociente de consumos.

Fuente: Desarrollado por Mayorga et al, para el Programa disminución de la carga de la enfermedad crónica no trasmisible en Colombia, 2014.

Se señala la orientación dada al cociente, visto como cociente de catetos. Justamente el cociente $\mathrm{t}_{\mathrm{ji}}$ corresponde a la tangente del ángulo $\mathrm{P}_{\mathrm{ji}}$ representado en el gráfico. De esta manera el valor del ángulo cuantifica el peso relativo que sobrelleva la familia en un rubro particular $\mathrm{R}_{\mathrm{i}}$ atribuible al cuidado del enfermo. Por lo tanto, un mayor ángulo tendrá una mayor tangente, es decir entre mayor sea ese peso relativo, mayor será el ángulo que lo representa ${ }^{18}$.

El cálculo del valor del ángulo, en grados, se concreta por medio de la función arco tangente. De esta manera,

$$
\mathrm{p}_{\mathrm{ji}}=\arctan \left(\frac{\mathrm{C}_{\mathrm{Xji}}}{\mathrm{C}_{\mathrm{Cji}}}\right), \mathrm{i}=1,2, \ldots, \mathrm{n}, \mathrm{j}=1,2, \ldots, \mathrm{k}
$$

Dado que el rango para cuantificar este peso relativo está entre 0 y 90 , como lo sugiere la figura 2 , un punto obligado de referencia en la lectura de sus valores es el ángulo de $45^{\circ}$ que representa una situación en la cual la cantidad de dinero que la familia dedica al cuidado del enfermo en un determinado rubro, es igual a la cantidad de dinero que la familia consume habitualmente en ese mismo rubro. Un ángulo mayor a éste significa que la cantidad de dinero que la familia dedica al cuidado del enfermo supera el consumo habitual de la familia en el respectivo rubro. Los puntos de referencia extremos $\left(0^{\circ} \mathrm{y}\right.$ $90^{\circ}$ ) significan que la familia no consume en el cuidado del enfermo en el primer caso o que el consumo es exclusivo para el cuidado del enfermo en el segundo caso. Dentro de la caracterización de la carga financiera atribuible al cuidado familiar del EC, dos atributos 
son imprescindibles en la descripción del peso relativo concerniente al rubro particular $\mathrm{R}_{\mathrm{j}}$ : el peso relativo medio y la desviación estándar, que se denotan como $\overline{\mathrm{p}}_{\mathrm{j}}$ y $\mathrm{S}_{\mathrm{pj}}$, cuyas expresiones son:

$\bar{p}_{j}=\frac{1}{n} \sum_{i=1}^{n} p_{j i}, \quad s_{p j}=\frac{1}{(n-1)} \sum_{i=1}^{n}\left(p_{j i}-\bar{p}_{j}\right)^{2}, j=1,2, \ldots, k$

Así entonces de manera sumaria, concurren el peso relativo medio y la desviación estándar concerniente al rubro particular $\mathrm{R}_{\mathrm{i}}$ tal como lo representa el triángulo ulterior (Figura 2).

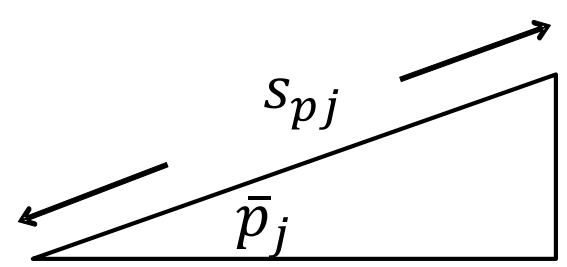

Figura 2. Triangulo ulterior en la cuantificación colectiva del peso relativo en el rubro $\mathrm{R}_{\mathrm{i}}$

Acá el ángulo representa el nivel medio y la hipotenusa la variabilidad del peso relativo del rubro $\mathrm{R}_{\mathrm{i}}$ en el conjunto de familias elegidas en la muestra. De esta forma, tomando en conjunto los rubros que incluye un análisis parcial o integral, los pesos relativos pueden compararse a partir del diagrama que compila en una sola unidad gráfica la distribución de los rubros respecto a su promedio y desviación estándar. El orden de colocación de los triángulos asemeja análogamente la formación de la concha de un caracol que va superponiendo capas en a modo de espiral.

\section{Declaración de aspectos éticos}

Se solicitó el consentimiento informado a los participantes, se contó con espacios adecuados para la aplicación de los instrumentos, se mantuvo la privacidad y confidencialidad, siguiendo las pautas éticas internacionales para la investigación 48 biomédica con seres humanos (CIOMS) ${ }^{19}$ y las normas éticas de la Declaración de Helsinki de 1975; se contó con la aprobación del Comité del Hospital Nacional de Ortopedia y Rehabilitación en donde se desarrolló el Protocolo.

\section{RESULTADOS}

En el estudio se identificaron las características de 30 diadas cuidador familiar-persona en EC. Se retomó el abordaje de Diada tal como es sugerido para el estudio del cuidado en situaciones de EC por cuanto da un mejor resultado que el individual y permite conocer al grupo familiar con una opción que apoya la focalización de los servicios de cuidado ${ }^{20}$.

El género de pacientes y cuidadores es mayoritariamente femenino con $73 \%$ de los pacientes y $80 \%$ de los cuidadores mujeres. Con respecto a la edad se encontró que $16.6 \%$ de los pacientes cuenta con menos de 20 años. De los 21 a los 40 hay 3,3\% de pacientes y $30 \%$ de los cuidadores, de los 41 a los $6016.6 \%$ de pacientes y $66.6 \%$ de los cuidadores y de 61 y más años $43,4 \%$ de los pacientes y $3.3 \%$ de los cuidadores. Estos datos coinciden con aquellos que afirman que la EC se incrementa con la edad ${ }^{20} \mathrm{y}$ que la mayor parte de las cuidadoras familiares son mujeres en edad intermedia que deben velar por su casa, su trabajo y sus seres queridos lo que les genera estrés y termina afectando su salud ${ }^{21}$.

Los diagnósticos médicos más frecuentes se asocian con trastornos cardiovasculares y metabólicos en los pacientes y se puede ver que los cuidadores presentan patologías propias de estrés físico o emocional que se han reportado con la actividad de ser cuidadores y que se conocen en algunos casos como el síndrome del cuidador ${ }^{22}$ (Tabla 1).

Con respecto a la escolaridad del paciente se encontró que $77.3 \%$ tienen un nivel educativo inferior a la primaria completa, un 16,6\% cuentan con un bachillerato parcial o total y el $9.1 \%$ con un nivel de educación superior técnico o profesional. Entre los cuidadores el 16,6\% tiene menos de primaria completa, el 23,4\% menos de un bachillerato completo y el $60 \%$ restante formación técnica o profesional. Puede verse que esta generación más joven que tiene al cuidado a sus mayores, cuenta con un nivel de estudio significativamente superior, tal como se presenta en el desarrollo educativo del país

En cuanto a la procedencia el 13,8\% de estas familias procede del sector rural mientras el $86.2 \%$ procede del urbano, con lo cual se encentra un grupo más citadino que la distribución nacional que refleja un $50 \%$ de población urbana en el país ${ }^{23,24}$. 
Tabla 1. Diagnósticos médicos de los integrantes de la diada persona con enfermedad crónica - cuidador familiar de Guatemala

\begin{tabular}{|l|c|c|}
\hline \multicolumn{1}{|c|}{ DIAGNÓSTICO MÉDICO } & PACIENTE \% & CUIDADOR \% \\
\hline ENFERMEDAD CEREBROVASCULAR (ECV) & 12,2 & 3,3 \\
\hline CÁNCER & 2,4 & 10,0 \\
\hline DM/TRANSTORNOS METABÓLICOS & 26,8 & 26,7 \\
\hline HTA & 24,4 & \\
\hline ALTERACIONES VISUALES Y CEGUERA & 2,4 & \\
\hline IRC & 4,9 & \\
\hline ENFERMEDAD PULMONAR & 7,3 & 13,3 \\
\hline ENFERMEDAD NEUROLÓGICA DIFERENTE & 17,1 & \\
A ECV & 0,0 & 53,3 \\
\hline TRASTORNO OSTEO MUSCULAR & 2,4 & \\
\hline SIN DIAGNÓSTICO DEFINIDO & & \\
\hline SANO & & \\
\hline
\end{tabular}

Nota: Algunos sujetos presentan más de una enfermedad.

Fuente: Datos del estudio, 2014.

El estado civil de los pacientes señala que el $30 \%$ cuentan con una pareja estable (20\% casados y $10 \%$ en unión libre). Mientras el 46,7 \% de los cuidadores tienen unión estable (36,7\% casados y 10\% unión libre). El 70\% de los pacientes y el $53.3 \%$ de los cuidadores no tienen compañero permanente.

Dentro de las ocupaciones de los pacientes se encuentran el $3.3 \%$ como empleados, el $10 \%$ estudiantes, el $56.7 \%$ dedicados al hogar, el 3.3\% jubilados, el 6.7\% hacen trabajos independientes y el $20 \%$ señalan tener otra condición sin poder precisar cuál es. Entre los cuidadores el $83.3 \%$ son empleados, ninguno estudia, el $3.3 \%$ se dedica al hogar, y el $13.4 \%$ trabaja de manera independiente.

Para estas familias el estrato socioeconómico es alto en el 3,3\%, medio en el $83,3 \%$, bajo en el $10 \%$ y el $3.3 \%$ no registró su nivel.

$\mathrm{Al}$ preguntar a los pacientes si ellos se perciben como carga para la familia se encontró que ninguno se percibe como una carga muy alta, el 13,3\% se percibe como una carga alta y el 3.3\% como una carga moderada, mientras el $66.4 \%$ perciben que son una carga baja para su grupo familiar.

De los cuidadores el $40 \%$ ha tenido experiencias previas de cuidado mientras el $60 \%$ cuida por primera vez.

Para terminar la caracterización de los cuidadores, se les valoró su nivel de percepción de carga con el cuidado. $46,7 \%$ señalaron tener ausencia de sobrecarga 43,3\% sobrecarga ligera y 10,0\% sobrecarga intensa.

Los medios de información y comunicación con que cuentan.

Cuando se auto valoró la apropiación de las Tecnologías de Información y Comunicación (TIC) por parte de las diadas se encontró que el cuidador tiene mayor apropiación con el teléfono (56,7\%) mientras el paciente lo tiene con la televisión $(53,3 \%)$ y el radio $(24,5 \%)$. Sin embargo, llama la atención que la mayoría no perciben las (TIC) como elemento de ayuda en el cuidado $(53,3 \%$ paciente y $76,7 \%$ cuidador). 


\section{Nivel de carga financiera familiar}

La metodología CARACOLA fue aplicada en la información obtenida con el $100 \%$ de las familias de Guatemala estudiadas para revisar integralmente el consumo real efectivo del grupo familiar en general (Tabla 2).

Percepción de la carga y consecuencias familiares. Con respecto a la percepción de carga se encontró que para estas familias el cuidado de su familiar genera una carga adicional. En el $88 \%$ de los casos, la familia debe asumir el costo extra. Aunque algunas tienen resuelta la situación económica al calificar el nivel de preocupación que les genera a las familias el desgate financiero debido al cuidado de la persona con enfermedad crónica, las participantes la calificaron como 1 sobre 4 en $27 \%$, en 2 sobre 4 el $50 \%$ en 3 el 15,4\% y en 4 o máxima preocupación posible el $7.7 \%$. Es decir, para el $23.1 \%$ la percepción de carga es alta o muy alta, en especial por tratarse de un consumo que se incrementa y se prolonga en el tiempo. En el 38.5\% de los casos la persona enferma asumía el manejo de las finanzas en el hogar mientras el 61,5 no lo hacía.

Tabla 2. Detalles de los diferentes rubros del consumo real efectivo de las familias asociado con el cuidado de una persona con enfermedad crónica

\begin{tabular}{|c|c|c|c|c|}
\hline COMPONENTE & NIVEL DE CARGA & DS & HIPOT. & RADIO PROMEDIO \\
\hline Alimentos & 13,9 & 15,0 & 15,44 & \\
\hline Comunicaciones & 19,0 & 9,7 & 10,30 \\
\hline Salud & 47,7 & 28,7 & 42,66 \\
\hline Transporte & 25,4 & 15,8 & 17,46 \\
\hline Vivienda & 19,9 & 17,0 & 18,04 \\
\hline \multicolumn{2}{|c|}{$\mathrm{n}=30$} \\
\hline
\end{tabular}

Fuente: Datos del estudio

El aspecto económico relacionado con el manejo de las finanzas por motivos del cuidado de la persona enferma, ha llevado a conflicto al interior del grupo familiar en el $23.1 \%$ de los casos; sin embargo, el $79.9 \%$ no han tenido conflicto. Aunque algunas familias señalan haber tenido que incurrir en préstamos, vender el automóvil, electrodomésticos y joyas para solventar los gastos del cuidado.

A continuación, se incluye el gráfico 50 del niel de carga percibido por las familias estudiadas con base en la aplicación de la metodología CARACOLA (Figura 3).

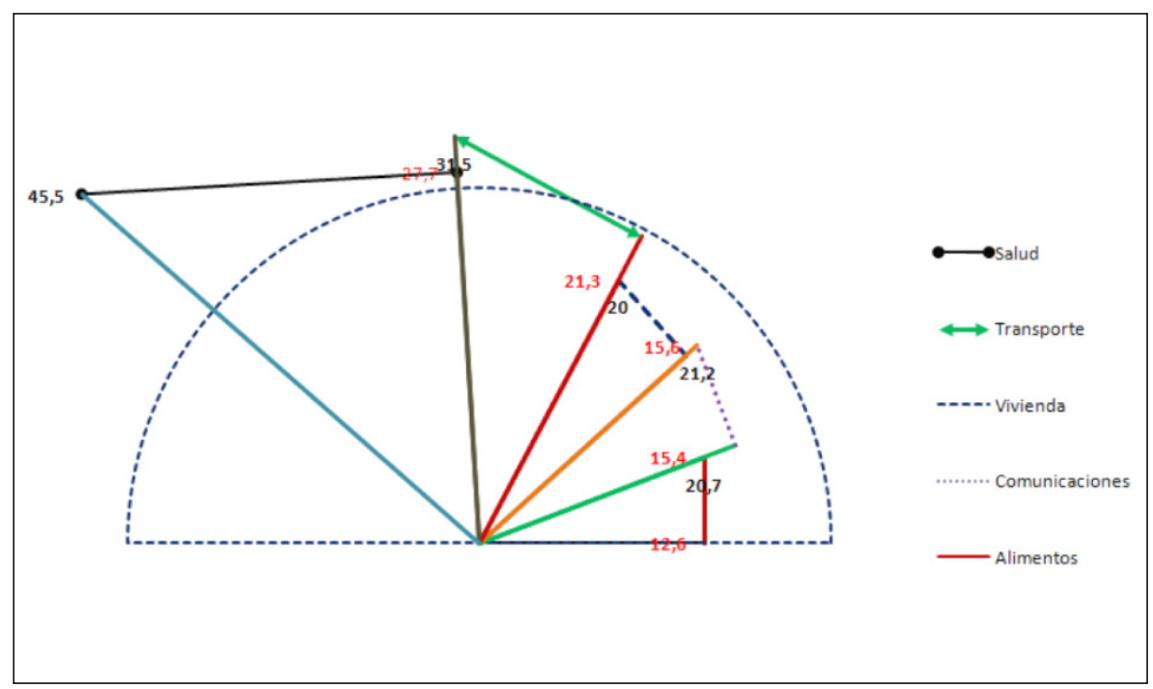

Figura 3. Carga de financiera familiar asociada con el cuidado de una persona con enfermedad crónica en Guatemala bajo la metodología CARACOLA 
En este se refleja que, en efecto, los gastos de salud y transporte son los que más agobian a las familias sin dejar de lado gastos de vivienda, comunicación, y alimentos, que todos se modifican a partir de la experiencia de cuidar a una persona con EC.

\section{DISCUSIÓN}

El estudio tomó familias que cuidan a personas con enfermedad crónica en Guatemala para revisar lo que sucede con respecto al costo familiar en esta experiencia en la vida cotidiana de estos grupos familiares ${ }^{12}$.

Los hallazgos reflejan la carga familiar relacionada con el consumo real efectivo generado por el cuidado de una persona con EC que ha sido ampliamente documentada por la literatura, pero hasta ahora no evaluada desde el punto de vista del cuidado de la persona enferma por parte de la familia y el consumo real efectivo familiar que esta situación exige ${ }^{25-28}$.

En el contexto guatemalteco sólo un estudio reciente se ha interesado por abordar la carga financiera ${ }^{29}$, señalando la estimación de los costos tangibles de la artritis reumatoide en términos de sus efectos en la productividad laboral y el impacto económico a nivel social, sin explorar el comportamiento de ésta carga a nivel familiar, lo que sí fue indagado en la presente investigación con el fin de contar con una perspectiva clara y objetiva que permita comprender las necesidades de las familias guatemaltecas en la vivencia del cuidado de un familiar con EC.

La mayoría de estudios relacionados con el costo de la enfermedad, demarcan su interés en macro-escenarios para obtener datos que revelen el estado actual del fenómeno y faciliten la toma de decisiones y la orientación de políticas económicas, sociales y sanitarias ${ }^{29-34}$, éste estudio permitió cuantificar hallazgos, revisar algunos aspectos cualitativos que reportan percepción de costos por parte de los usuarios ${ }^{1}$, generar una análisis que apoya la comunicación de un esfuerzo hasta ahora no abordado de los modelos, para comparar y medir tendencias, momentos, escenarios, estrategias de disminución de consumo o fortalecimiento de esfuerzos ${ }^{35,36}$.

La metodología permitió determinar el esfuerzo financiero adicional en la experiencia del cuidado de la salud desde la perspectiva de la familia discriminando los gastos que se asocian con servicios de salud de aquellos que se vinculan directamente al consumo familiar señalando que unos y otros hacen parte de la misma carga del cuidado ${ }^{37-39}$.

\section{CONCLUSIÓN}

Los hallazgos muestran la mayor carga alrededor de los gastos propios de la salud, el transporte, la vivienda, las comunicaciones y los alimentos, en ese orden.

La carga financiera de las familias guatemaltecas que cuidan una persona con EC estudiadas, medida con la encuesta "Costo financiero del cuidado de la Enfermedad crónica”, y analizada bajo la metodología CARACOLA es alta y tiene un impacto significativo en la familia. Este estudio respalda la recomendación de revisar e incluir en las políticas públicas la forma de considerar y mitigar el consumo real efectivo del grupo familiar que afronta esta situación.

\section{DECLARACIÓN SOBRE CONFLICTO DE INTERESES}

Los autores no reportan conflictos a declarar relacionados con la investigación.

\section{AGRADECIMIENTOS}

A las familias que voluntariamente quisieron participar de este estudio por todos sus aportes para comprender mejor la carga financiera del cuidado familiar de una persona con enfermedad crónica. Al Grupo de Cuidado al Paciente Crónico de la Universidad Nacional de Colombia por su respaldo en este trabajo como parte de la Red Latinoamericana del Cuidado al Paciente Crónico.

\section{REFERENCIAS BIBLIOGRAFICAS}

1. Organización Mundial de la Salud. Estadísticas sanitarias mundiales. Ginebra; 2013.

2. Organización Mundial de la Salud. Diseases Country Profiles. Informe técnico. Ginebra; 2014.

3. Piamjariyakul U, Yadrich DM, Russell C, Myer J, Prinyarux C, Vacek JL, et al. Patients' annual income adequacy, insurance premiums and out-of-pocket expenses related to heart failure care. Heart Lung. 2014 Sep-Oct;43(5):469-75.

4. Luczak J, Garcia-Gómez P. Financial burden of drug expenditures in Poland. Health Policy. 2012; 105(2-3): 256-64. 
5. Tucker PS, Kingsley MI, Morton RH, Scanlan AT, Dalbo VJ.The Increasing Financial Impact of Chronic Kidney Disease in Australia. Int J Nephrol. 2014;2014: 1-7.

6. Chaves OCS, Silveira AD, Predebon JCA, Ibaldo SDS. You have to take care": family's experiences and knowledge/ patient caregiver with chronic disease. Journal of Nursing UFPE. 2015; 9(10); 9535-9540. DOI: 10.5205/01012007.

7. Daivadanam M. Pathways to catastrophic health expenditure for acute coronary syndrome in Kerala. ¿Good health at low cost?. BMC Public Health. 2012; 12(306):1-8

8. Brinda EM, Rajkumar AP, Enemark U, Attermann J, Jacob,KS. Cost and burden of informal caregiving of dependent older people in a rural Indian community. BMC Health Serv Res. 2014;14(207):2-9.

9. Murphy A, Mahal A, Richardson E, Moran AE. The economic burden of chronic disease care faced by households in Ukraine: a cross-sectional matching study of angina patients. Int J Equity Health. 2013; 12(38): 1-8.

10. Dantés H, Castro V, Franco F, Bedregal P, Rodriguez J, Espinoza A, et al. Burden of disease in Latin America. Salud Publica Mex. 2011; 53(2):s72-s77.

11. Lozano R, Gómez H, Franco M, Rodriguez A. Carga de la enfermedad en municipios urbanos marginados (20042008). En: BID, Observatorio de la Salud para América Latina y el Caribe, Funsalud. México; 2009.

12. Velásquez A. Guía Metodológica para las Estimaciones Epidemiológicas del Estudio de Carga de Enfermedad. Lima: Abt Associates Inc; 2006.

13. Barrera L, Pinto N, Sánchez B, Carrillo G, Chaparro L. Cuidando a los Cuidadores. Bogotá: Universidad Nacional de Colombia; 2010.

14. Prada SI, Takeuchi Y, Ariza Y. Costo monetario del tratamiento de la enfermedad de Alzheimer en Colombia. Acta Neurol Colomb. 2014; 30(4): 247-255.

15. Contreras AA. Costo financiero del cuidado de una persona con enfermedad crónica: preocupaciones de los cuidadores familiares. [Tesis de pregrado en Enfermería, modalidad de pasantía dentro del Programa para la Disminución de la carga de la ECNT en Colombia]. Bogotá: Universidad Nacional de Colombia; 2014.

16. Sánchez B, Chaparro L, Carrillo G. Encuesta de caracterización del cuidado de la diada cuidador familiar - persona con enfermedad crónica. Rev. cien. cuidad. 2014; 11(2): 31-45.

17. Montoya LA, Montoya I, Ocampo J, Sánchez B, Chaparro L. Diseño y validación de la encuesta "Costo financiero del cuidado de la enfermedad crónica no transmisible" en Colombia. Salud Uninorte. En prensa; 2016.

18. Mayorga H, Montoya LA, Montoya I, Sánchez B, Sandoval N. Metodología para analizar la carga financiera del cuidado familiar de una persona con enfermedad crónica. Rev.cienc.biomed. 2015;6(1):96-106.
19. Consejo de organizaciones internacionales de las ciencias médicas. Pautas éticas internacionales para la investigación biomédica en seres humanos preparadas por el consejo de organizaciones internacionales de las ciencias médicas. Ginebra: Consejo de organizaciones internacionales de las ciencias médicas; 2012.

20. Chaparro L. Cómo se constituye en el "vínculo especial" de cuidado entre la persona con enfermedad crónica y el cuidador familiar. Aquichán. 2011; 11(1):7-22.

21. Pérez Jiménez D, Rodríguez Salvá A, Herrera Travieso DM, García Roche R, Echemendía Tocabens B, Chang de la Rosa M. Caracterización de la sobrecarga y de los estilos de afrontamiento en el cuidador informal de pacientes dependientes. Revista cubana de higiene y epidemiologia. 2013; 51(2): 174-183.

22. Ruiz MAV, de la Cruz García C, Navarrete RAM, Hernández CMM, Reyna M. D. Cansancio, cuidados y repercusiones en cuidadores informales de adultos mayores con enfermedades crónico degenerativas. European Journal of investigation in health, psychology and education. 2015 4(2):151-160.

23. Dueñas E, Martínez M, Morales B, Muñoz C, Viáfara A. Syndrome of the caregiver in disability old patients and the psychosocial implications, Colombia 2003-2004. Colombia Médica 2009;37(2):31-38.

24. Ministerio de educación de Guatmala. Políticas de calidad educativa y alfabetización. 2015 [Internet]. [Consultado 2015 Nov 10]. Disponible en: http://www. conalfa.edu.gt/queescona2.html.

25. The worldfact book. Datos de urbanización Guatemala. [Internet]. [Consultado 2015 Dic 18]. Disponible en: https://www.cia.gov/library/publications/the-worldfactbook/geos/gt.html

26. Onukwugha E, McRae J, Kravetz A, Varga S, Khairnar R, Mullins CD. Cost-of-Illness Studies: An Updated Review of Current Methods.PharmacoEconomics. 2016;34(1): 43-58.

27. Azevedo LF, Costa-Pereira A, Mendonça L, Dias CC, Castro-Lopes JM. (2016). The economic impact of chronic pain: a nationwide population-based cost-ofillness study in Portugal. The European Journal of Health Economics, 17(1), 87-98.

28. Celano CM, Healy B, Suarez L, Levy DE, Mastromauro C, Januzzi JL, et al. Cost-Effectiveness of a Collaborative Care Depression and Anxiety Treatment Program in Patients with Acute Cardiac Illness. Value in Health. 2016; 19(2):185-191.

29. García García CO. Costo social y económico de la artritis reumatoide. [Tesis doctoral en salud pública]. Guatemala:Universidad de San Carlos de Guatemala; 2015. 
30. Costa N, Derumeaux H, Rapp T, Garnault V, Ferlicoq L, Gillette S, et al. Methodological considerations in cost of illness studies on Alzheimer disease. Health economics review; 2012: 2(1): 1-12.

31. Doria A, Amoura Z, Cervera R, Khamastha MA, Schneider M, Richter J.. Annual direct medical cost of active systemic lupus erythematosus in five European countries. Annals of the rheumatic diseases.2014; 73(1): 154-160.

32. Angelis A, Tordrup D, Kanavos P. Socio-economic burden of rare diseases: A systematic review of cost of illness evidence. Health Policy. 2015;119(7): 964-979.

33. de Oliveira ML, Santos LMP, da Silva EN. Direct healthcare cost of obesity in Brazil: An application of the cost-of-illness method from the perspective of the public health system in 2011. PloS one. 2015; 10(4): e0121160.

34. Seuring T, Archangelidi O, Suhrcke M. he economic costs of type 2 diabetes: a global systematic review. Pharmacoeconomics.2015; 33(8): 811-831.

35. Anderson JL, Heidenreich PA, Barnett PG, Creager MA, Fonarow GC, Gibbons RJ, et al. ACC/AHA statement on cost/value methodology in clinical practice guidelines and performance measures: a report of the American College of Cardiology/American Heart Association Task Force on Performance Measures and Task Force on Practice Guidelines. Journal of the American College of Cardiology.2014; 63(21): 2304-2322..

36. John J, Wolfenstetter SB, Wenig CM. An economic perspective on childhood obesity: recent findings on cost of illness and cost effectiveness of interventions. Nutrition. 2012; 28(9): 829-839.

37. Raftery M, Ryan P, Normand C, Murphy A, de la Harpe D, McGuire B. The Economic Cost of Chronic Noncancer Pain in Ireland: Results From the PRIME Study, Part 2. The Journal of Pain. 2012; 13(2):139-145.

38. Olesen J, Gustavsson A, Svensson M, Wittchen HU, Jönsson B.. The economic cost of brain disorders in Europe. European Journal of Neurology.2012; 19(1): 155-162.

39. Dee A, Kearns K, O’Neill C, Sharp L, Staines A, O’Dwyer $\mathrm{V}$, et al. The direct and indirect costs of both overweight and obesity: a systematic review. BMC research notes. 2014;7(1): 242.

Para citar este artículo: Morales-Padilla ON, Pinituj-Monroy GA, Escobar-Vásquez CA. Carga financiera del cuidado del enfermo crónico en un grupo de familias en Guatemala. Duazary. 2017 enero; 14 (1): 45 - 53 